EXTENDED REPORT

\title{
Expression of growth differentiation factor- 5 and bone morphogenic protein-7 in intraocular osseous metaplasia
}

\author{
S Toyran, A Y Lin, D P Edward
}

Br J Ophthalmol 2005;89:885-890. doi: 10.1136/bjo.2004.056374

See end of article for authors' affiliations

......................

Correspondence to:

Deepak P Edward, MD,

Department of

Ophthalmology, University

of Illinois at Chicago, 1905

W Taylor Street, L217,

Chicago, IL 60612, UŚA;

deepedwa@uic.edu

Accepted for publication 1 December 2004

\begin{abstract}
Background/aims: Intraocular bone is seen in a wide spectrum of ocular disorders. The pathogenetic mechanisms of bone formation in the eye are unclear. Growth differentiation factor-5 (GDF-5), bone morphogenic protein-7 (BMP-7), and transforming growth factor beta-1 (TGF $\beta 1$ ) are multifunctional cytokines that have important roles in bone formation. Immunohistochemistry was used to localise GDF-5, BMP-7, and TGF $\beta 1$ in the human eye to determine their role in intraocular bone formation.

Methods: Paraffin embedded sections from human eyes included fetal eyes $(n=5)$, normal adult eyes $(n=4)$, eyes with osseous metaplasia $(n=8)$, and eyes with focal fibrous metaplasia of the retinal pigment epithelium (RPE) without osseous metaplasia $(n=2)$. Immunohistochemistry was performed using indirect immunofluorescence with antibodies to GDF-5, BMP-7, and TGF $\beta 1$. The staining intensity was evaluated semiquantitatively in the RPE, retina, ciliary epithelium, and cornea; and analysed statistically.

Results: When compared with normal adult eyes, which showed no RPE immunoreactivity, the RPE metaplasia surrounding areas of osseous metaplasia showed mild GDF-5 and moderate BMP-7 $(p=0.004)$ intracytoplasmic immunoreactivity. In contrast, trace GDF-5 and mild BMP-7 staining was seen in zones of RPE fibrous metaplasia in areas not associated with osseous metaplasia. Mild intracytoplasmic TGF $\beta 1$ expression was seen in the RPE metaplasia surrounding the bone when compared with adult eyes. Both fetal and adult eyes showed trace to mild GDF-5 and BMP-7 labelling of the non-pigmented ciliary epithelium which was increased in the eyes with osseous metaplasia. In eyes with osseous metaplasia, a significant decrease in GDF-5 and BMP-7 labelling was noted in fetal keratocytes ( $p=0.0159$ for both antibodies) when compared to adult eyes. Also, a significant decrease in BMP-7 labelling was seen in keratocytes in eyes with osseous metaplasia $(p=0.0162)$.

Conclusions: The increase in GDF-5, BMP-7, and TGF $\beta 1$ immunoreactivity in zones of RPE metaplasia in eyes with osseous metaplasia suggests that these proteins have an important role in intraocular ectopic bone formation.
\end{abstract}

ntraocular bone is seen in a many ocular disorders, including longstanding retinal detachment, chronic inflammation, and phthisis bulbi. ${ }^{1-4}$ Bone morphogenic proteins (BMPs) are multifunctional cytokines, members of the transforming growth factor beta (TGF $\beta$ ) superfamily of growth factors that have a wide range of effects that include regulation of cell growth, differentiation, and apoptosis. ${ }^{5}$ In the eye they are expressed in the cornea, ciliary epithelium, lens epithelium, retina, and blood vessels. ${ }^{7}$ These proteins act as autocrine and/or paracrine factors regulating bone growth and remodelling. ${ }^{8}$

Ectopic ossification originates from osteoprogenitor stem cells lying dormant within the affected soft tissues. With the proper stimulus, the stem cells differentiate into osteoblasts and lead to osteoid formation, eventually leading to mature ectopic bone.

Although many isoforms of BMP induce bone formation, BMP-7 is a potent osteoprogenitor. Growth differentiation factor-5 (GDF-5) has an important role in long bone development in humans, and has been shown to induce differentiation of mesenchymal cells into osteoblast lineages. ${ }^{9}$ Both BMP-7 and cartilage derived morphogenetic protein-1 (also known as GDF-5) are overexpressed in pleomorphic adenomas of the salivary gland where heterotopic bone formation may be seen. ${ }^{10}{ }^{11}$ These findings suggest GDF-5 and BMP-7 may have important roles in extraosseous bone formation. To evaluate this hypothesis in the eye, we examined the expression of GDF-5 and BMP-7 by immunohistochemistry in normal adult and fetal human eyes, and in eyes with intraocular osseous and fibrous metaplasia.

\section{MATERIALS AND METHODS}

Sections from enucleated specimens were obtained from the archives of the pathology laboratory of the University of Illinois at Chicago, IL, USA. The project was approved by the institutional review board at the University of Illinois at Chicago and complied with the Declaration of Helsinki. Sections from human eyes included fetal eyes $(n=5)$ (range of age 57-72 days), normal adult eyes $(n=4)$, eyes with osseous metaplasia in the posterior pole $(n=8)$, and eyes with focal fibrous metaplasia of the retinal pigment epithelium (RPE) without osseous metaplasia $(n=2)$. Eyes with RPE fibrous metaplasia and tumour were excluded. A hip bone tissue specimen was used as a positive control. The specimens were fixed in $10 \%$ buffered formalin, processed, and embedded in paraffin wax. The tissue with bone had been previously decalcified.

\section{Immunohistochemistry}

Immunohistochemistry was performed on deparaffinised slides using indirect immunofluorescence technique. Before incubation with primary antibody, antigen retrieval with sodium citrate buffer ( $\mathrm{pH}$ 6.0) was performed. The primary antibodies used are described in table 1 . The reaction product was visualised using $\mathrm{Cy} 3$ conjugated streptavidin solution and the nuclei counterstained with DAPI.

Abbreviations: BMP-7, bone morphogenic protein-7; GDF-5, growth differentiation factor-5; RPE, retinal pigment epithelium; TGF $\beta 1$, transforming growth factor beta-1 


\begin{tabular}{lllll|}
\hline \multicolumn{2}{l}{ Table 1} & Primary antibodies \\
\hline Antibody & Type & Dilution & Catalogue No & Source \\
\hline GDF-5 & Polyclonal & $1: 100$ & Sc-6901 & Santa Cruz Biotechnology, Inc (Santa Cruz, \\
BMP-7 & Polyclonal & $1: 100$ & Sc-6899 & Santa Cruz Biotechnology, Inc \\
TGF- $\beta$ 1-G & Polyclonal & $1: 25$ & Sc-146-G & Santa Cruz Biotechnology, Inc \\
\hline
\end{tabular}

\section{Data analysis}

The immunolabelled specimens were examined under a Zeiss microscope, using Axioskop 2 Plus software. Two independent observers assigned a semiquantitative score to each slide based on the intensity of staining: negative $=0,1+($ trace $)=$ $1-25,2+($ mild $)=26-50,3+($ moderate $)=51-75,4+$ (intense) $=76-100$. A Mann-Whitney non-parametric test was used to compare the staining intensity between normal adult, fetal, and osseous eyes.

\section{RESULTS}

The results of staining for GDF-5 and BMP-7 in the normal adult, fetal and osseous eyes are shown in figures 1 and 2 . Adjacent sections stained with haematoxylin and eosin are presented for orientation. Figure 3 shows TGF $\beta 1$ immunoreactivity in an eye with osseous metaplasia. Figure 4 shows the staining intensity of normal adult, fetal, and osseous eyes in the RPE, retina, ciliary epithelium, and cornea.

\section{Retina and retinal pigment epithelium (RPE)}

GDF-5 and BMP-7 immunoreactivity was absent in the normal adult sensory retina and RPE (figures not shown). In fetal eyes mild GDF-5 (fig 1A, B) and BMP-7 (fig 1C, D) labelling was present in Muller cell processes. In eyes with osseous metaplasia, mild GDF-5 (fig 1E, F) and moderate BMP-7 (fig $1 \mathrm{G}, \mathrm{H}$ ) intracytoplasmic immunoreactivity was seen, especially in areas of RPE fibrous metaplasia surrounding bone. Staining was less intense in the osteoblasts. Trace GDF-5 and BMP-7 immunoreactivity was seen in the retina.
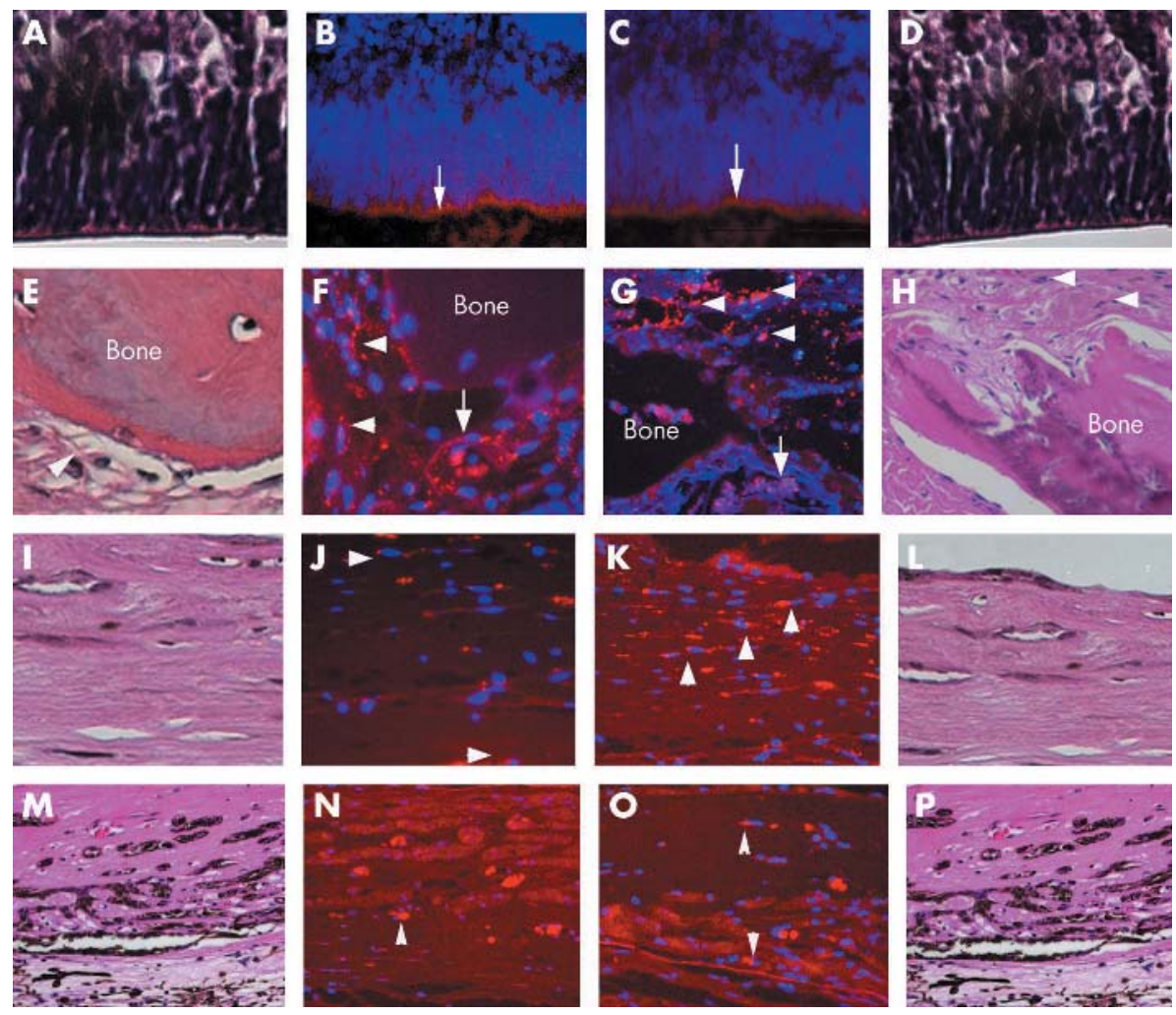

Figure 1 Immunostaining of the retina and RPE. Cy3 is the fluorophore (red) and DAPI is the nuclear counterstain (blue). (A) Fetal retina (haematoxylin and eosin; magnification $\times 40$ ). (B) Mild GDF-5 staining of Muller cell processes of the retina in (A) (arrow) (magnification $\times 40$ ). (C) Mild stippled BMP-7 staining of Muller cell processes of the fetal retina in (D) (arrow) (magnification $\times 40$ ). (D) Fetal retina (haematoxylin and eosin; magnification $\times 40$ ). (E) Area of osseous metaplasia surrounded by a zone of RPE fibrous metaplasia (arrowhead) (haematoxylin and eosin; magnification $\times 20$ ). (F) Mild to moderate stippled GDF-5 staining of RPE fibrous metaplasia around the bone in (E) (arrowheads). Bruch's membrane is indicated by the arrow (magnification $\times 20$ ). (G) Moderate stippled BMP-7 staining of RPE metaplasia (arrowheads) in $(H)$ around the bone. Bruch's membrane is indicated by the arrow (magnification $\times 20$ ). (H) Area of osseous metaplasia surrounded by RPE fibrous metaplasia (arrowheads) (haematoxylin and eosin; magnification $\times 20$ ). (I) RPE fibrous metaplasia distant from the area of bone formation (haematoxylin and eosin; magnification $\times 20$ ). (J) Trace GDF-5 staining of RPE fibrous metaplasia in (I) (arrowheads) (magnification $\times 20$ ). (K) Mild BMP-7 staining of RPE fibrous metaplasia in (L) (arrowheads) (magnification $\times 20$ ). (L) RPE fibrous metaplasia distant from the area of bone formation (haematoxylin and eosin; magnification $\times 20$ ). (M) RPE fibrous metaplasia in an eye without bone formation. (haematoxylin and eosin; magnification $\times 20$ ). (N) Trace to mild GDF-5 staining of RPE fibrous metaplasia in (M) (arrowhead) (magnification $\times 20$ ). (O) Trace to mild BMP-7 staining of RPE fibrous metaplasia in (P) (arrowheads) (magnification $\times 20$ ). (P) RPE fibrous metaplasia in an eye without bone formation (haematoxylin and eosin; magnification $\times 20$ ). 

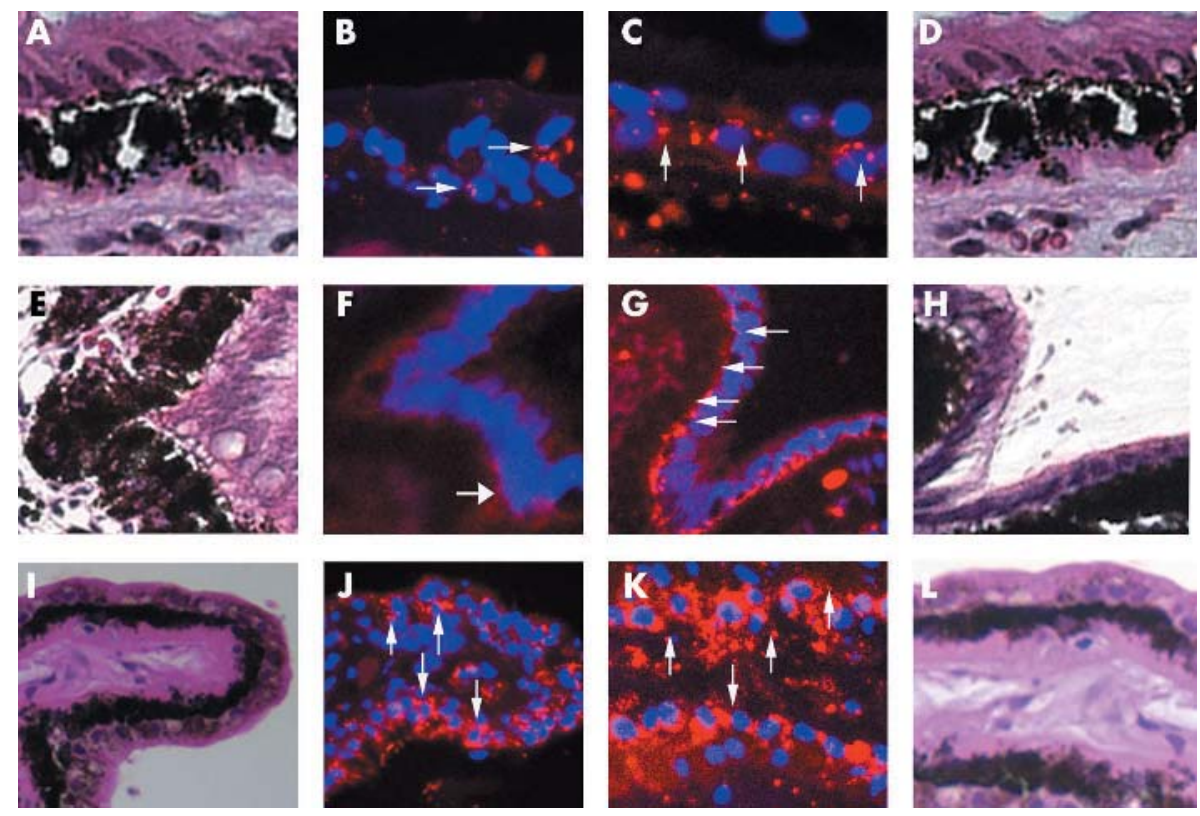

Figure 2 Immunostaining of the non-pigmented ciliary epithelium. (A) Ciliary epithelium of the normal adult eye (haematoxylin and eosin; magnification $\times 40$ ). (B) Mild stippled GDF-5 staining of the non-pigmented ciliary epithelium in (A) (arrows) (magnification $\times 40$ ). (C) Mild stippled BMP-7 staining of the non-pigmented ciliary epithelium in (D) (arrows) (magnification $\times 40$ ). (D) Ciliary epithelium of the normal adult eye (haematoxylin and eosin; magnification $\times 40$ ). (E) Ciliary epithelium of the fetal eye (haematoxylin and eosin; magnification $\times 40$ ). (F) Trace stippled GDF-5 staining of the non-pigmented ciliary epithelium in (E) (arrow) (magnification $\times 40$ ). (G) Moderate BMP-7 staining of the non-pigmented ciliary epithelium in (H) (arrows) (magnification $\times 40)$. (H) Ciliary epithelium of the fetal eye (haematoxylin and eosin; magnification $\times 40$ ). (I) Ciliary epithelium of the eye with osseous metaplasia (haematoxylin and eosin; magnification $\times 20$ ). (J) Mild to moderate diffuse GDF-5 staining of the nonpigmented ciliary epithelium in the eye with the osseous metaplasia in (I) (arrows) (magnification $\times 20$ ). (K) Moderate diffuse BMP-7 staining of the nonpigmented ciliary epithelium in the eye with the osseous metaplasia in (L) (arrows) (magnification $\times 40$ ). (L) Ciliary epithelium of the osseous metaplasia eye (haematoxylin and eosin; magnification $\times 40$ ).

In addition, trace GDF-5 (fig $1 \mathrm{I}, \mathrm{J}$ ) and mild BMP-7 (fig $1 \mathrm{~K}$, L) immunoreactivity was seen in zones of RPE fibrous metaplasia not associated with osseous metaplasia. Trace GDF-5 (fig 1M, N) and BMP-7 (fig 1O, P) immunoreactivity was seen in zones of RPE fibrous metaplasia of the eye without osseous metaplasia.

TGF $\beta 1$ immunoreactivity was absent in normal adult and fetal eyes. Mild intracytoplasmic TGF $\beta 1$ (fig 3) immunoreactivity was seen in the RPE surrounding the bone. TGF $\beta 1$ immunoreactivity was only evaluated in the RPE and areas of RPE metaplasia.

Only the increased intensity of BMP-7 labelling in eyes with osseous metaplasia was statistically significant (significance versus normal; $\mathrm{p}=0.004$ ) (fig $4 \mathrm{~A}, \mathrm{~B}$ ).

\section{Ciliary epithelium}

There was mild intracytoplasmic staining of GDF-5 and BMP7 in the non-pigmented ciliary epithelium of normal adult eyes (fig 2A, B and 2C, D). GDF-5 staining in the fetal eye was less intense than in normal non-pigmented ciliary epithelium (fig 2E, F). However, BMP-7 immunoreactivity was more intense than in normal adult eyes (fig 2G, H). In eyes with osseous metaplasia, moderate GDF-5 (fig 2I, J) and BMP-7 (fig 2K, L) expression was noted in the nonpigmented ciliary epithelium and faint BMP-7 immunoreactivity was seen in the pigmented ciliary epithelium. Moderate GDF-5 immunoreactivity was seen in the stromal cells of the ciliary body.

The differences in expression of GDF-5 and BMP-7 in fetal ( $\mathrm{p}=0.4127, \mathrm{p}=0.5556, \mathrm{GDF}-5$ and BMP-7 respectively) and osseous eyes $(\mathrm{p}=0.3677)$ compared to normal eyes did not reach statistical significance (fig $4 \mathrm{C}$ ).

\section{Cornea}

Moderate GDF-5 and BMP-7 staining was noted in keratocytes of normal adult eyes (figures not shown). Fetal keratocytes showed trace labelling with both antibodies. The decreased GDF-5 and BMP-7 immunoreactivity in fetal keratocytes when compared to normal eyes was statistically significant ( $\mathrm{p}=0.0159$ for both antibodies). Mild staining of
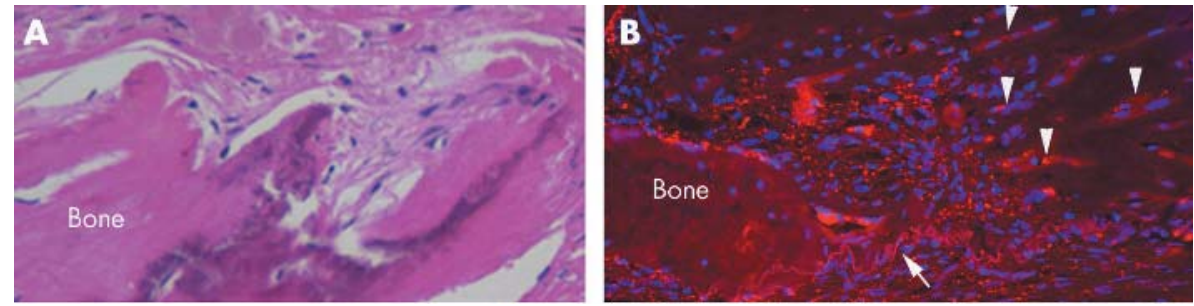

Figure 3 Immunostaining of the RPE with TGF $\beta 1$. (A) RPE fibrous metaplasia surrounding bone (haematoxylin and eosin; magnification $\times 20$ ). (B) Mild to moderate stippled TGF $\beta 1$ staining of RPE metaplasia (arrowheads) around the bone in (A). Bruch's membrane is indicated by the arrow (magnification $\times 20$ ). 

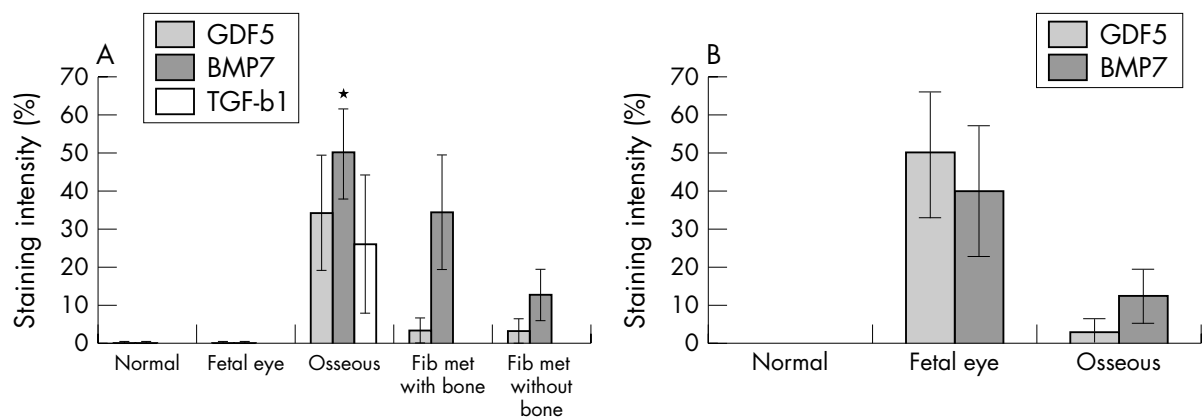

Figure 4 Staining intensity of GDF-5 and BMP-7 in (A) RPE; (B) retina; (C) ciliary epithelium; and (D) cornea of normal adult and fetal eyes, and eyes with osseous metaplasia. The staining intensity of TGF $\beta 1$ is only evaluated in the RPE (A), and includes eyes with RPE fibrous metaplasia without bone formation.
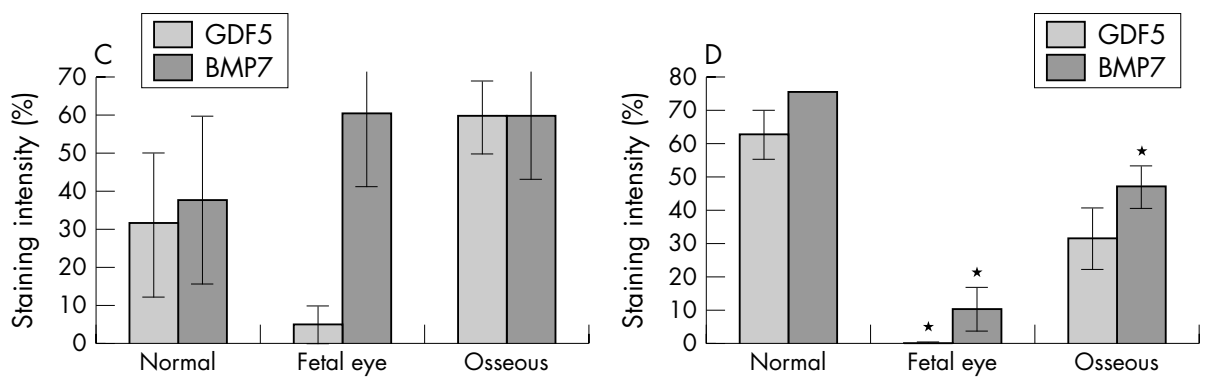

GDF-5 and BMP-7 was observed in keratocytes of eyes with osseous metaplasia. Only the decrease in BMP-7 expression was significantly compared to normal eyes $(p=0.0162)$ (fig 4D).

\section{DISCUSSION}

Ectopic ossification is the abnormal formation of bone within extraskeletal soft tissues. Some BMPs (BMP-2-8, 13, 14) are potent inducers that aid in transforming undifferentiated mesenchymal cells into cartilage and bone forming elements. Of these, BMP-7 is a potent and well described osteoprogenitor and it is theorised that cytokines like BMP-7 induce ectopic ossification by inducing differentiation of pluripotential cells, mesenchymal cells, or fibroblasts into osteoprogenitor cells. ${ }^{11-13}$

Intraocular bone formation usually occurs in the setting of a chronically diseased eye. Ectopic ossification may occur as a result of local or systemic inflammation. Local pathology like chronic retinal detachment, hyperplasia, and transdifferentiation of RPE appear to be a few of the prerequisites for intraocular osseous metaplasia. ${ }^{14}$

Furthermore, pathological studies have shown that ocular inflammation is a common finding in eyes with bone formation. ${ }^{14}$ The inflammatory infiltrate usually consists of chronic inflammatory cells including monocytes and macrophages, which produce cytokines such as interleukin-1 (IL-1) and tumour necrosis factor alpha $(\mathrm{TNF}-\alpha){ }^{15}$

Although the presence of IL-1 or TNF- $\alpha$ was not directly investigated in the current study, it may have an indirect role in bone formation through activation of other cytokines. TNF- $\alpha$ and IL- 1 are reported to stimulate activated cells to produce TGF $\beta 1^{16}$ and BMP-7. ${ }^{17}{ }^{18}$ It is possible that in the presence of inflammation, similar events may occur in the RPE, which is known to express TGF $\beta 1 .{ }^{19}$ TGF $\beta 1$ promotes the transformation of epithelial cells to mesenchymal cells in the kidney ${ }^{20}$ and possibly promotes the transformation of normal RPE into fibroblasts (RPE fibrous metaplasia), which is a common finding in eyes with osseous metaplasia. In our study eyes with osseous metaplasia had increased TGF $\beta 1$ expression in areas of RPE metaplasia when compared to normal eyes. Bosse et al demonstrated TGF $\beta 1$ mRNA in areas of mesenchymal tissue proliferation, and proposed that TGF $\beta 1$ was a local cellular regulator of ectopic bone formation. ${ }^{21}$ Although the increase in TGF $\beta 1$ immunoreactivity was not statistically significant because of small numbers, the study in general supports its role in ectopic bone formation.

BMP-7 stimulates the transformation of mesenchymal cells into osteoblasts. In the eye, ectopic bone formation is mainly seen in areas of RPE fibrous metaplasia. ${ }^{14}$ In our study there was significant BMP-7 immunoreactivity in areas of RPE fibrous metaplasia surrounding bone. In contrast, eyes with RPE fibrous metaplasia without osseous metaplasia showed a mild increase in BMP-7 staining compared to normal eyes. This suggests that the expression of BMP-7 needs to reach a threshold level before the fibrous metaplasia cells transform into osteoblasts, which might explain the fact that all chronic ocular inflammatory disorders do not result in bone formation.

Additionally, BMP-7 inhibits the transformation of epithelial cells into fibroblasts. ${ }^{20}$ Since BMP-7 was detected in metaplastic RPE cells and not in normal RPE, the metaplastic RPE may be inhibiting the transformation of normal retinal pigment epithelial cells into fibrous cells, a process that was promoted by TGF $\beta 1$. Co-localisation of BMP-7 and TGF $\beta 1$ to areas of fibrous metaplasia suggests that these cytokines have an important role in regulating the transformation of RPE into fibrous cells and bone.

We therefore propose the following model for bone formation inside the eye (fig 5). Chronic end stage eye disease is often accompanied by intraocular inflammation. The inflammatory cells release IL- 1 or TNF- $\alpha$, stimulating the RPE to produce TGF $\beta 1$ and BMP-7. TGF $\beta 1$ triggers epithelial-mesenchymal transformation of RPE cells into RPE fibrous metaplasia. BMP-7 inhibits this transformation by counteracting the effect of TGF $\beta 1$. Additionally, BMP-7 promotes the transformation of metaplastic RPE into osteoblasts. It is likely that GDF-5, which was co-localised with BMP-7 in areas of RPE metaplasia, also stimulates osseous metaplasia. In rats, GDF-5 has been shown to induce the formation of cartilaginous tissue as well as bone. ${ }^{22}$ GDF-5 was found in osteoblast-like cells from the primary ossification centres of long bones. ${ }^{23}$ 


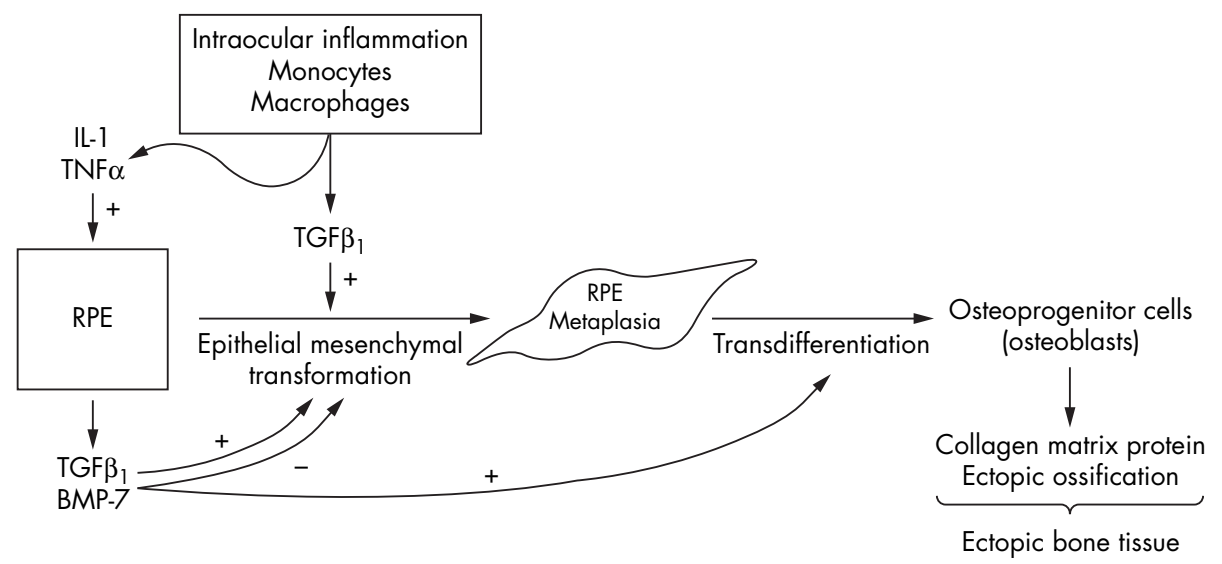

Figure 5 Schematic diagram with proposal pathways of intraocular bone formation. Inflammatory cells synthesise interleukin (IL-1), tumour necrosis factor (TNF- $\alpha$ ), and transforming growth factor beta-1 (TGF $\beta 1$ ). IL-1 and TNF- $\alpha$ stimulate retinal pigment epithelium (RPE) to produce TGF $\beta 1$ and bone morphogenic protein-7 (BMP-7). Both TGF $\beta 1$ released from RPE and inflammatory cells trigger epithelial-mesenchymal transformation (from RPE cells to RPE metaplastic cells). BMP-7 balances these effects by inhibiting this process. On the other hand, increased BMP-7, if excessive, can cause transdifferentiation from RPE metaplasia to osteoblasts.

It is likely that these cytokines exercise their effects on target cells through ligand receptors. In rat eyes, TGF-beta type I and II receptors and BMP type IA, IB, and II receptors are expressed in the RPE, and other ocular structures. ${ }^{24}$ BMP-7 binds predominantly to BMP type IB receptor in the rat osteoprogenitor-like cell line. ${ }^{25}$ Also receptor binding studies of GDF-5 revealed that GDF-5 has affinity for the BMP receptor type IA, IB, and II. ${ }^{26}$

Though this model of intraocular ossification focused on inflammatory stimuli causing bone formation, intraocular bone formation has been well recognised in non-inflammatory proliferations such as teratomas, medulloepitheliomas, and choroidal osteomas. Our study did not specifically address these conditions; however, overexpression of cytokines such as GDF-5 and BMP-7 are reported in other neoplasias such as pleomorphic adenomas of the salivary gland, which are sites for heterotopic bone formation..$^{10}$

Although the expression of BMP-7 in adult and fetal human eyes has not been previously studied, focal mRNA expression has been detected in animal embryos. ${ }^{27}{ }^{28}$ BMP-7 has been shown in chick embryo $\mathrm{RPE}^{29}$ and developing mouse RPE. ${ }^{30}$ In our study fetal and normal adult RPE failed to show BMP-7 immunoreactivity. BMP-7 and GDF-5 immunoreactivity was absent in the normal adult sensory retina. BMP-7 has been shown to induce dendritic growth in rat sympathetic neurons. ${ }^{31}$ However, since dendritic growth presumably is not a prominent feature of normal, mature human retina, one would expect that BMP-7 is not highly expressed. In animal embryos, BMP-7 is abundantly expressed at the junction between the neuroepithelium and RPE (likely Muller cell processes). ${ }^{27-29}$ Our findings confirm this expression pattern in human tissue.

The expression of BMP-7 in the non-pigmented ciliary epithelium of human fetal eyes confirms previous studies that showed moderate BMP-7 expression in the nonpigmented ciliary epithelium of late stage mouse embryos and postnatal mice. ${ }^{32}$ It was postulated that BMP-7 was required for ciliary body development. In contrast, there was decreased expression of GDF-5 compared to normal eyes, suggesting that GDF-5 may not have a role in ciliary body development. These changes are not statistically significant, probably because of the small sample size. Although not statistically significant, there was a trend towards increased expression of BMP-7 and GDF-5 in the non-pigmented ciliary epithelium of eyes with osseous metaplasia. This may result from inflammation in the ciliary body or diffusion of cytokines (for example, IL-1) from the posterior pole through the vitreous, stimulating the ciliary epithelium to produce BMP-7 and GDF-5.

Moderate BMP-7 and GDF-5 immunoreactivity was seen in keratocytes of normal adult corneas. BMP-7 and GDF-5 are expressed in human keratocytes ${ }^{32}$ and may be important regulators of corneal epithelial cell proliferation. ${ }^{33}$ A significant decrease in the expression of GDF-5 and BMP-7 was observed in the corneas of fetal eyes. GDF-5 inhibits the proliferation of corneal epithelial and stromal cells. ${ }^{33}$ It may need to be downregulated for proper corneal development. Alternatively, factors required for corneal development might downregulate GDF-5 and BMP-7. Or, perhaps these proteins are not important for corneal development.

Decreased expression of GDF-5 and BMP-7 was also observed in corneas with osseous metaplasia compared to normal eyes; however, the decrease was only significant for BMP-7. The reason for the decreased expression of BMP-7 in the cornea is not clear, since the cornea is not directly affected by the ossification.

In summary, we propose a potential pathway for the formation of bone within the eye that suggests that BMP-7 has an important role in this process. It is possible that other BMPs and cytokines not investigated in this study also have a role in this complex process. GDF-5 also appears to have a role in osseous metaplasia but further studies may help understand the signalling pathway for bone formation and better define the role of GDF-5. In addition, this study confirmed in human tissue some of the previously published expression patterns of BMP-7 and GDF-5 in animals.

\section{ACKNOWLEDGEMENTS}

A gift from William Pendill Glaucoma Research Foundation.

\section{Authors' affiliations}

S Toyran, D P Edward, Department of Ophthalmology and Visual Sciences, University of Illinois at Chicago, Chicago, IL, USA

A Y Lin, D P Edward, Department of Pathology, University of Illinois at Chicago, Chicago, IL, USA

This work was supported by NEI Core Grant for Vision Research (EY 01792) supported by Research to Prevent Blindness, Bethesda, MD, USA.

Competing interests: none declared 


\section{REFERENCES}

1 Finkelstein EM, Boniuk M. Intraocular calcification and hematopoesis. Am J Ophthalmol 1969;68:683-90.

2 Monselise M, Rapaport I, Romem M, et al. Intraocular ossification. Ophthalmologica 1985;190:225-9.

3 Evans NM. Ocular ossification-a case report and review of literature. Trans Ophthalmol Soc U K 1986;105:330-6.

4 Shields CL, Shields JA, Augsburger JJ. Choroidal osteoma. Surv Ophthalmol 1988;33:17-27.

5 Wordinger RJ, Agarwahl R, Talati M, et al. Expression of bone morphogenetic proteins (BMP), BMP receptors and BMP associated proteins in human trabecular meshwork and optic nerve head cells and tissues. Mol Vis 2002;8:241-50.

6 Reddi AH. Bone and cartilage differentiation. Curr Opin Genet 1994; 4:737-44.

7 Yamashita H, Tobari I, Sawa M, et al. Functions of the transforming growth factor-beta superfamily in eyes. Nippon Ganka Gakkai Zasshi 1997; 101:927-47.

8 Centrella M, Horowitz MC, Wozney JM, et al. Transforming growth factor family members and bone. Endocr Rev 1994;15:27-39.

9 Yamaguchi A, Komori T, Suda T. Regulation of osteoblast differentiation mediated by bone morphognetic proteins, hedgehogs, and Cbfal. Endocr Rev 2000;21:393-411.

10 Kusafuka K, Yamaguchi A, Kayano T, et al. Expression of bone morphogenetic proteins in salivary pleomorphic adenomas. Virchows Arch 1998:432:247-53.

11 Kusafuka K, Luyten FP, De Bondt R, et al. Immunohistochemical evaluation of cartilage-derived morphogenic protein-1 and -2 in normal human salivary glands and pleomorphic adenomas. Virchows Arch 2003;442:482-90.

12 Gruber R, Kandler B, Fuerst $G$, et al. Porcine sinus mucosa holds cells that respond to Bone morphogenetic protein (BMP)-6 and BMP-7 with increased osteogenic differentiation in vitro. Clin Oral Implants Res 2004;15:575-80.

13 Rutherford RB, Moalli M, Franceschi RT, et al. Bone morphogenetic protein transduced human fibroblasts convert to osteoblasts and form bone in vivo. Tissue Eng 2002;8:441-52.

14 Vemuganti GK, Honavar SG, Jalali S. Intraocular osseous metaplasia. A clinico-pathological study. Indian J Ophthalmol 2002;50:183-8.

15 Dayer JM. Evidence for the biological modulation of IL-1 activity: the role of IL-1 Ra. Clin Exp Rheumatol 2002;20(5 Suppl 27):14-20.

16 Chao CC, Hu S, Sheng WS, et al. Tumor necrosis factor-alpha mediates the release of bioactive transforming growth factor-beta in murin microglial cell cultures. Clin Immunol Immunopathol 1995;77:358-65.

17 Merrihew C, Soeder S, Rueger DC, et al. Modulation of endogenous osteogenic protein -1 (OP-1) by interleukin-1 in adult human articular cartilage. J Bone Joint Surg Am 2003;85-A(Suppl 3):67-74.
18 Yoshida S, Kubota Y, Toba T, et al. Induction of osteogenic protein-1 expression by interleukin-1 beta in cultured rabbit articular chondrocytes. Tohoku J Exp Med 2002; 197:101-9.

19 Tanihara $\mathbf{H}$, Yoshida M, Matsumoto $M$, et al. Identification of transforming growth factor- $\beta$ expressed in cultured human retinal pigment epithelial cells. Invest Ophthalmol Vis Sci 1993;34:413-19.

20 Zeisberg M, Kalluri R. The role of epithelial to mesenchymal transition in renal fibrosis. J Mol Med 2004;82:175-81.

21 Bosse A, Wulf M, Wiethege T, et al. Collagens and growth factors in heterotopic ossification. Pathologe 1994;15:216-25.

22 Hotten GC, Matsumoto T, Kimura M, et al. Recombinant human growth/ differentiation factor 5 stimulates mesenchyme aggregation and chondrogenesis responsible for the skeletal development of limbs. Growth Factors 1996; 13:65-74.

23 Chang SC, Hoang B, Thomas JT, et al. Cartilage derived morphogenetic proteins. New members of the transforming growth factor beta superfamily predominantly expressed in long bones during human embryonic development. J Biol Chem 1994;269:28227-34.

24 Obata H, Kaji Y, Yamada H, et al. Expression of transforming growth factorbeta superfamily receptors in rat eyes. Acta Ophthalmol Scand 1999;77:151-6.

25 Tamaki K, Souchelnytskyi S, Itoh S, et al. Intracellular signaling of osteogenic protein-1 through Smad5 activation. J Cell Physiol 1998;177:355-63.

26 Erlacher L, McCartney J, Piek E, et al. Cartilage-derived morphogenetic proteins and osteogenic protein-1 differentially regulate osteogenesis. J Bone Miner Res 1998; 13:383-92.

27 Dudley AT, Lyons KM, Robertson EJ. A requirement for bone morphogenetic protein-7 during development of the mammalian kidney and eye. Genes Dev 1995;9:2795-807

28 Trousse F, Esteve P, Bovolenta P. BMP4 mediates apoptotic cell death in the developing chick eye. J Neurosci 2001;21:1292-301.

29 Vogel-Hopker A, Momose T, Rohrer H, et al. Multiple functions of fibroblast growth factor-8 in chick eye development. Mech Dev 2000;94:25-36.

30 Hung FC, Zhao S, Chen Q. Retinal ablation and altered lens differentiation induced by ocular overexpression of BMP-7. Vis Res 2000;42:427-38.

31 Horbinski C, Stachowiak EK, Chandrasekaran V, et al. Bone morphogenetic protein-7 stimulates initial dendritic growth in sympathetic neurons through an intracellular fibroblast growth factor signaling pathway. J Neurochem 2002;80:54-63.

32 Zhao S, Chen Q, Hung FC, et al. BMP signaling is required for development of the ciliary body. Development 2002;129:4435-42.

33 You L, Kruse FE, Pohl J, et al. Bone morphogenetic proteins and growth and differentiation factors in the human cornea. Invest Ophthalmol Vis Sci 1999:40:296-311. 